\title{
Review: dynamic exercise increases aerobic capacity and muscle strength in rheumatoid arthritis
}

\author{
van den Ende CH, Vliet Vlieland TP, Munneke M, et al. Dynamic exercise therapy in rheumatoid arthritis: a systematic review. Br J \\ Rheumatol 1998 Jun;37:677-87.
}

\section{Question}

Can dynamic exercise therapy improve joint mobility, muscle strength, aerobic capacity, and daily functioning without increasing adverse effects in patients with rheumatoid arthritis (RA)? Dynamic exercise was defined as exercise of sufficient intensity to improve muscle strength and aerobic capacity.

\section{Data sources}

English, Dutch, French, or German language trials were identified using Medline (1964-97), Embase/Excerpta Medica (1974 to October 1996), SCISEARCH (1974 to October 1996), and Russmed Articles (1988 to October 1996) with the terms rheumatoid arthritis, exercise therapy, motion therapy, physical education and training, and gymnast: (with various endings). Bibliographies were checked and authors were contacted.

\section{Study selection}

Randomised controlled trials were selected if they compared dynamic exercise therapy with another form of exercise therapy or a non-exercising control group; participants had confirmed RA using the 1958 or 1987 American Rheumatism Association criteria; interventions were designed to improve aerobic capacity, muscle strength, or both; the session frequency was $\geqslant$ twice/week; duration of the programme was $\geqslant 6$ weeks; each session had the potential for heart rate to exceed $60 \%$ of maximal heart rate for $\geqslant 20$ minutes; and outcomes were aerobic capacity, muscle strength of the knee extensors, joint mobility, functional performance (15 m walk test), functional ability (Health Assessment Questionnaire or Arthritis Impact Measurement Scales or another validated health related quality of life tool), self reported pain, or disease activity (sedimentation rate or swollen joint count).

\section{Data extraction}

Data were extracted on study quality (10 criteria), patient information (age, sex, disease duration, and inclusion and exclusion criteria), intervention description and duration, and outcomes with assessment methods.

\section{Main results}

6 studies were included. The number of participants ranged from 18-100 (median 32). Mean age in 5 studies was approximately 50 years. 4 studies included patients with stable medications. 4 studies excluded patients with moderate to severe disability. The exercise programme lasted $\leqslant 3$ months in 5 studies. 5 programmes included bicycle exercises. Many of the programmes were multifaceted.

Data were not pooled because of differences in study methods and outcomes, but were reported as per cent change from baseline. 5 studies measured aerobic capacity, and 3 showed improvement in the dynamic exercise group compared with the control group. 4 studies measured muscle strength; in 2 studies the patients in the dynamic group increased their strength. 2 studies evaluated joint mobility and both showed improved mobility, although only 1 reached statistical significance. 3 studies used the walk test; 1 showed an improvement with dynamic exercise. 5 studies measured functional abilities; none showed improvements. Disease activity and pain were assessed in several trials and none showed differences between the dynamic exercise and control groups.

\section{Conclusion}

Dynamic exercise increases aerobic capacity and muscle strength in patients with rheumatoid arthritis, but does not lead to differences in disease activity or pain.

Source of funding: no external funding.

For correspondence: $\operatorname{Dr}$ C H M van den Ende, Netherlands Institute of Primary Care, P O Box 1568 , 3500 bn Utrecht, the Netherlands. Fax +31302729729.

\section{Commentary}

Aerobic capacity of patients with RA is frequently reduced with pain, stiffness, muscle weakness, and loss of range of joint movement leading to limited functional capacity. ${ }^{12}$ Aerobic exercise has been shown to be beneficial across a range of rheumatological conditions, particularly when the disease is in remission or the joints are relatively inactive. Studies have shown that exercise can improve function without triggering a flare up and also can reduce pain, depression, and anxiety. ${ }^{34}$ The systematic review by van den Ende et al addresses these problems and is therefore a useful addition to knowledge of the subject.

Because strict inclusion and exclusion criteria were used, only studies of patients with less severe, established disease were reviewed. This limits the generalisability of the results given the chronic, progressive, cyclical pattern of RA. The data were comprehensively reviewed and presented, however, giving the reader access to a complex set of related studies. Methodologically stronger trials show less positive results than weaker trials. Given these limitations, it would be useful for further research to look at the benefits of dynamic exercise in patients with early RA across a wider range of functional capacity and to determine whether improved long term outcomes occur in all patients.

For the individual patient, probably the most important factors are pain and level of disability. Patients frequently question nurses about the benefits and detrimental effects of exercise. This study gives nurses the evidence they need to inform and reassure patients that vigorous, dynamic exercise can improve fitness without increasing pain.

Elizabeth M Barrett, RGN, RHV, MSc Clinical Research Manager St Michael's Hospital Norfolk, UK

1 Ekblom B, Nordermar R. Rheumatoid arthritis. In: Skinner JS, editor Exercise testing and exercise prescription for special cases. Philadelphia: Lea and Febiger, 1987:101-14.

2 Minor MA, Hewett JE, Webel RR, et al. J Rheumatol 1998;15:905-11.

3 Semble EL, Loeser RF, Wise CM. Semin Arthritis Rheum 1990;20:32-40.

4 Stenstrom CH. Arthritis Care Res 1994;7:190-7. 